\title{
Mediterranean Energy Dream and Cultural Planning between Old Layers and New Paradigms
}

\author{
Emanuela $\operatorname{Nan}^{1 \text { a }}$ \\ ${ }^{1}$ Via Nizza 2/5 Genoa 16145, Italy \\ aemanuela.nan@gmail.com
}

Keywords: Mediterranean Territory, Sharingswitch, Rinaturactivation, Upcycling.

\begin{abstract}
In the recent years that the interconnection of the production, distribution and use of energy cannot be considered unconnected to the materials and information chains. A global solution to the problem of energy supply has to be looked for in a new approach taking into account all these factors in dependence of the local resources and characteristics (geographic, urban, cultural etc) of the territories. In areas such as the Mediterranean, where the ability to tap into the huge renewable and clean energy resources, are confronted with the reality of contexts secularly layered and saturated, in which, as perhaps nowhere else, landscapes and scenery of quality and value environmental and town are mixed and interwoven with situations of degradation, worthlessness and abandonment, the reorganization energy is, in this sense, an incredible opportunity to rethink and redefine space and scenarios.
\end{abstract}

\section{Introduction}

In the post-globalization era the energy issue has become a major challenge. The increasing awareness of the strong effects of traditional energy resources use on environment and climate, combined with their increasing scarcity, requires the elaboration of new political, technological and socio-economic approaches aimed at the definition of more sustainable energy and materials means of production, distribution and use. These approaches center on the proper design of an integrated network of energy and materials production chains based on the concept of renewability, eco-compatibility and sustainability.

In fact, it has become clear in the recent years that the interconnection of the production, distribution and use of energy cannot be considered unconnected to the materials and information chains. A global solution to the problem of energy supply has to be looked for in a new approach taking into account all these factors in dependence of the local resources and characteristics (geographic, urban, cultural etc) of the territories. New equilibrium has to be designed to satisfy the generally increasing energy request while preserving as much as possible ecology and sustainability. The main challenge in designing a harmonic development roadmap has to face with the complexity of the multiple interconnection among these different facets and of their complex interactions. A natural approach to the solution of this problem require the set-up of an effective network of knowledge, technologies and awareness distributed in the involved communities and properly adapted to the local requirements and pre-existences. The concept of localization of the problem implies the definition of the proper intervention scales capable of addressing the request of small communities as well as of larger urban sites and industrialized regions. New modality of production, requiring different technologies and economic structures, distribution, favoring the creation of an effective network among communities, and use of energy and materials have to be defined. The definition of the most appropriate technologies and a careful evaluation of their advantages and drawbacks represent the basic motivation of this research program.

Mediterranean basin countries, for their geographical and climate location, as well as for their asymmetrical development stages and socio-economical situations, would be particularly adapt to the experimentation of new approaches to the energy and materials issues. However, even if this topic is 
now widely discussed all around the world, Mediterranean regions do not seem to fully exploit their potentials in the field.

The main reason for this lack of initiatives seems indeed to rely on the same socio-economical conditions of these territories. While the South Mediterranean countries are particularly blocked by an unstable socioeconomic and political situation, the Northern countries, even if technologically advanced, have to deal with regions characterized by a largely built, stratified, widespread and often saturated heritage and with an economy strongly linked to the traditional energy resources use. Nevertheless, according to the 2011 GSE statistical report (Energy Services Manager) [1], Spain and Italy have reached the second and third place in Europe for the production of renewable energy sources (RES). This simple observation reveals the real possibilities of the Mediterranean region, if they will be fully converted to a renewed approach to sustainable energy, they could become a global exemplar area for a new territorial, socio-economic and political model. A feasible strategy to reach this goal has to be designed by determination of diversified and coordinated approaches for the two Mediterranean regions aimed to overcome their respective deficits. The Northern side has to establish new modalities of relationships, exchange and organization, taking in account both the larger dimensions of an extended scenario including south Mediterranean countries and modified local and global levels of interfacing of heterogeneous entities, pointing toward an increasingly relevance of "green economy" in their socio-economical policies. They have, although, to cross individual interests, immediate solutions to prune their benefits to a larger scale in terms of space and time. In the southern region, in reverse, a consolidated and disseminated energy production system does not exist yet, so it is possible to structure, from the start, territories and energy networks in a sustainable way, but adequate economic resources and technological expertise are still lacking.

The optimal reorganization, from energetic point of view, of the Mediterranean region has to aim at a synergic cooperation of the individual parts in favor of the entire area. In this sense, it is required a more democratic vision where the local regains its relevance. The networks and connections speed exceeded the traditional concepts of space-time; every reality, as part of a system, can play a major role expressing its full potential and sharing its benefits, receiving what it needs from the others. Accordingly, a careful evaluation and enforcement of new schemes of energy production, distribution and use, taking advantage of a network organization among Mediterranean countries, must also take in account materials and information production and distribution chains.

\section{North and south mediterranean energy dement ions}

Nowadays European countries cover approximately $70 \%$ of energy demand in the Mediterranean Region. But this scenario will change in the next future due to the growth of the south Mediterranean countries and to the population increase that will drastically change the current situation with epoch making impact on the future demand for energy and infrastructure needs. In the next future it is expected an increase in energy demand in the Mediterranean countries of about $1.5 \%$ per year by 2030. According to a recent valuation of the OME (Observatories Mediterranean of Energy) [2], the energy annual growth rates of the south countries will reach a percentage of $42 \%$ of the total energy use of the entire Mediterranean area by 2030 .

To meet the new energy requirements, the role of the renewable energies will be as important as that of the traditional energy sources (gas, coal, oil). As matter of facts, many research and development programs have been launched in the past recent years in the field of solar and wind power generation technologies. Examples of these efforts are the projects Deserted, Tran green/Med Grid, CoCoNet active in the framework of the Mediterranean Solar Plan (MSP) started by the Union for the Mediterranean (UfM) in 2008 that is also economically supported by EU. The analysis of the total current production of energy in the Mediterranean countries shows that the energy demand in this geographical area amount to about 7.6 TWh per year representing 5\% of the world total demand compared with $9.6 \%$ of China, $13.7 \%$ of ex URSS countries and $12.9 \%$ of Middle East demand. Gas, coal and oil still represent more than two thirds of energy global production in the Mediterranean. 
However, in the last three decades, many changes occurred in the distribution of power sources relevance. As a result of the increasing relevance of the other energy sources, even if the main source of energy is still the oil and its consumption slightly increases in absolute value of $0.29 \%$ per year, its percentage reduced from $59 \%$ to $38 \%$. Oil production is concentrated above all in Algeria (42\%), Libya (42\%) and Egypt (16\%). The reduction in energy production from oil has been more than compensated by an increase of gas production of 2,3\% per year from 1980 to 2007 making this source the second most relevant in the Mediterranean area. The main producers are Algeria (53\%), Egypt $(33 \%)$ and Libya (10\%).

A recent analysis of International Energy Agency (IEA) showed that the major producers of thermal energy from coal (7\% of total energy production) are Turkey and Greece, followed by Spain and Serbia. Nuclear energy represents the third source of energy with the production of about 1,5 TWh in France, Slovenia and Spain (reaching a significant 20\% of total energy production). Renewable energies represent about $10 \%$ of the total offer. Actually North Mediterranean countries hold the most part of the production. It is expected in the next few year a significant increase of the production of energy from renewable sources in the South-West countries, particularly in Egypt. Among the renewable energy sources, in addition to hydropower, biomass and waste represent about $6 \%$ of the total production. However, biomass contribution is underestimated as it does not consider the significant amount of wood used mostly for domestic heating and cooking that is a very widely used energy source in Morocco and Tunisia.

As regards to wind power, North Africa countries promoted in the past few years numerous initiatives to develop this energy production means: In 2008, Egypt started a plan for the generation of electricity from wind farms that should rise the percentage of energy produced from wind to $12 \%$ of country demand by 2020; Libya should increase the production of energy from wind farms to reach a total potentiality of $1 \mathrm{GW}$ by installing new plants for an amount of least $500 \mathrm{MW}$. Being the only north African country without relevant fossil fuels resources Morocco started an action to reduce its dependence upon coal and oil import by increasing the amount of energy produced from wind. The objective is to cover $14 \%$ of national energy demand by this means by 2020 . To this aim there are currently under construction wind farms totaling a power of $1.3 \mathrm{GW}$. Tunisia is also pushing on the realization of many wind farms that should cover up to $20 \%$ of the national power demand. Algeria is also developing wind production plants. This country is also involved in the realization of an electric power line to be realized along the MEDGAZ gas pipeline. This realization could represents a key contribution to the realization of an electric power network between Europe and North Africa.

In this context, characterized by a huge number of initiatives and a general address toward the development of renewable energy source plants, there are still many technical, economical and political barrier that obstacle the full development of the renewable sources market in and among Mediterranean countries. Nevertheless, the social and environmental benefits that a strong development of such technologies could produce represent a strong motivation to their development.

In particular, Northern and southern Mediterranean shores countries should seek a shared policy aimed to the definition of a network for the exchange of knowledge, competences and technologies capable of foster the birth of an energy market and to promote and rationalize the commercial and technological exchanges among involved countries. These countries could play in such a market the roles of supplier and users depending on their natural and technological resources in a self-balanced and profitable way promoting a more harmonic development of Mediterranean area.

\section{New cultural planning}

The saturation and stratification of contemporary urban territories added to the speed of the changes brought about by instances, fashions, trend development of social, political, economic ... ever new and ever changing, has madding it increasingly difficult to define intervention programs and operations planning on a larger scale. 
Although these processes are ongoing for some time and the administration and governance at all levels should seek to adapt continually defining and newly developed tools for faster and more flexible action, starting from the 90 s town planning is becoming strategy planning, the ever increasing speed of change of the boundary conditions and the increased exponentially of the variables involved continues to highlight the need for more targeted approaches and clear horizons, dividing independently shares and function.

In essence, today the planning seems to be working primarily through the identification of future scenarios and strategies operational times not to determine, but to coordinate a set of projects and actions of intervention on the cities and territories.

This condition, combined with the increasing scarcity of resources due to the economic crisis and to imposing a renewed ecological awareness, produces the emergence of a whole system of new paradigms which recognize their own definition in the use of the suffix re - : re-cycle, re-activation, re- naturalization, re-connect, ...

The interesting aspect of the emergence of these logics is the substantial re-breaking and re-versing these underlying trend with respect to the consolidated implementation and continuous addition typical zoning and planning of the operations of the recent past.

In this sense, the scenarios are re-interpreted.

\section{Logic of Stress $>$ Logic of Empathies}

The return to nature desire inevitably introduces changes in the relationship between solids and voids turning the green from being just a urban complement, infiltrated and often almost accidental, in an important and articulated organization and space-time modulation device able to modulate it, to interact and interface with weight and percentage surface to the built.

The strengthened natural system not only becomes a potential alternative to the infrastructure system in favor of a pedestrian mobility, bicycle or any other alternative to the car, but in a way even more interesting, it assumes the role of a reliever and a diaphragm replacing limits and tensions for empathy and exchange between the parties.

The urban limit, however, is dissolving completely and permanently in the landscape and in the same way the boundary between public space and building, in terms of area and permeability, tends to dissolve.

Hybridization, in this view, seems to impose itself strongly as a new way of resolving spatial and formal disputes, mingling and overlapping, prefiguring urban realities made of multi-purpose convertible and usable spaces and systems, at the same time in a different way, and objects as buildings, infrastructure, social spaces and energy sources.

\section{Logic of Agreements > Logic of Resonances}

The revaluation of each single reality enriches the territory defining it as a set of independent and, at the same time, consistent and concurrent specificities in the definition of the set.

This constitutes an important shift in territory design and action because it inserts and gives role also to minor realities, while the already worthy recognized areas are, in this way, unmarked, freed by an excessive pressure, which redistributes itself in adjacent areas, recovering breath and quality.

Constantly changing, the logical dynamics clarification and understanding involve the choice and the proposition of directions able to determine and drive the renewal and the interface between different territory's devices, outlining new configurations, prepositions, evolving and alternatives geographies of landscape.

In this logical context, the landscape takes shape, therefore, in new geographies no longer tied to the traditional categories of natural and artificial or urban and agricultural [3]. In this new dimension, the territory founds these natures mixed and overwritten and, at the same time, distinguishes and organizes itself respect to their valences:

- Propulsive, as a generator of new arrangements, configurations; 
- Potential, as reinforcement of situations and processes already in place;

- Connective, as an articulating binder not only at physical level, but also cognitive;

- Suspensive, as a pause or a buffer [4].

Basically, we are witnessing the gradual establish and codify of a contrast way to the densification at all costs and to the indifference to the asset values ( ecological, landscape, architectural ,...) [5]. You go to an awareness of the impossibility to procrastinate further actions to rebalance and correction of destructive and speculative processes following the first industrial revolution and increased, more and more alarming, with the development of technologies and industrialization.

This new approach gives rise not only to new and ever more complex models of strategic planning, but, most interestingly, it will define and give value to a whole system of territories (networks of urban spaces and not ) so far ignored considered marginal or waste, but instead are being rediscovered as potential engines of new models and horizons of development and the city (or cities) in a sustainable way.

\section{Trands from urban mediterranean territories}

The Mediterranean is a unique setting and a kaleidoscopic melting pot; a calm, protected basin which thanks to its central position has for centuries been a crossroads connecting three continents; a place for meeting and exchange.

The Mediterranean coasts possess an accumulation of exceptional urban places that are uniquely rich, layered and complex, and whose physical and geographical features have enabled them to play a fundamental role in the development of civilisations. The port cities around the Mediterranean are highly sophisticated artefacts of major heritage value and are the outcome of a process dating back to the beginning of time but also expressing the varied, many-sided present day as well as looking forward to the future [6]. But the complex connotations of this urban fabric cannot simply be explained as a consequence or a product of the interaction between the morphology of the Mediterranean territory, the evolution of a single civilisation, or the result of applying different rules. More interestingly, they are the end product of contaminations and cross-fertilisations that were mainly spontaneous and peaceful and that came about thanks to the contacts, exchanges and interchanges between the various populations and cultures of the basin. Because of these processes, which took place over time and across cultures, each Mediterranean port city developed its own identity and each one is completely different from the others, whilst at the same time being undeniably and indissolubly connected to them as an inextricable part of a whole system.

The Mediterranean port cities, which are linked not merely by formal similarities (or by their similarities of form), but with respect to a number of key concepts (their interactions, exchanges, dependencies) in a more underlying, intrinsic way, can be identified and defined using a logical analogy according to which as the system of the Mediterranean basin they are something like a biological organism. In this different perspective they reveal themselves to be perfect space-systemic workshops whose greater or lesser complexity of interconnectedness defines the Mediterranean itself as a relational phenomenological model that embodies, in summary form, the logics and relationships of the global network as a whole [7].

The special condition of this context, in the new dimension of attention to energy development of cities and territories, highlights new ways to define spaces. In particular three trends emerge:

- Rinaturactivation, as recovery of that relationship-interdependence between settlements and territories. The Mediterranean urbanity developed for centuries in harmony with its territory by exploiting the morphology and optimizing the available resources in a manner calculated to achieve maximum energy efficiency with less effort and waste.

- Sharingswitch, as mutual correlation between the different spaces and areas of the city and territory into a game for activation and deactivation constantly exchange flows and energy. 
- Upcycling, as recovery of ancient uses and spaces with new meanings and roles in relation to both the definition that the operation of the city and the territory.

On the one hand because of their structural complexity, their internal mixed, and their strong interactivity within (and with respect to) the basin as a system, and on the other because of their strong desire to affirm themselves and be protagonists within (and with respect to) the global level, the Mediterranean territories show that without any loss of meaning they can fully intercept and absorb the various logical and use-related modifications imposed by today's dynamics and equally, in this process, can reveal that in response to the new demands and stresses they are able to suggest, develop, and propose paradigmatic scenarios and tactics that are expressed in the conjunction, conversion, harmonization and completion of the existing, whether or not it has heritage value.

\section{References}

[1] GSE is the state-owned company which promotes and supports renewable energy sources (RES) in Italy. In particular, GSE fosters sustainable development by providing support for renewable electricity (RES-E) generation and by taking actions to build awareness of environmentally-efficient energy uses

[2] OME is a nonprofit Association created in 1988. The Association counts thirty two leading Mediterranean energy companies from fourteen countries. The offices are located in NanterreFrance. The main objective of the Association is to promote cooperation and collaboration with major energy companies operating in the Mediterranean region, making of energy an element for regional integration

[3] "Cities happen to be problems in organized complexity, like the life sciences. They present situations in which half a dozen of several dozen of quantities are all varying simultaneously and in subtly interconnected ways...the variables are many, but they are not helter skelter; they are inter-related into an organic whole" Jacobs J.: The Death and Life of Great American Cities, Random House and Vintage Books, New York (1961)

[4] E. Nan: Dynamic Geographies of Desire's Territories, in Planum n. 2, Vol. 27/2013

[5] In this scenario arises the Landscape Urbanism. The term Landscape Urbanism appears for the first time, as a branch of Landscape Ecology that focuses on the organization of human activities into the natural landscape (Shane 2004), the exhibition Landscape Urbanism (1997) edited by Charles Waldheim. It posed to light the study of the interrelationships between human activities and the natural landscape: the interstitial spaces, the spaces infrastructure and ecology are considered as the background of the social activities planned and unplanned on public land. It is therefore a model of performative urbanism (Corner 1997), or performing practice (Shane 2005), as an analysis of the development of cities attentive to the needs of the reconstitution of the delicate ecological balance between the built and unbuilt. The thorny problem of how urban density emerges from the landscape and how the urban ecologies and technologies define the spaces in which case the Company's business is dealt with according to a bottom-up approach

[6] The literature on the Mediterranean is very large, in particular with regard the complexity of the scenarios are the reference works are Matvejevic' P.: Breviario mediterraneo, Garzanti, Milano (1991) and Braudel F.: Il Mediterraneo. Lo spazio e la storia, gli uomini e la tradizione, Milano, Bompiani, (1987)

[7] J. F. Troin: Le Metropoli del Mediterraneo. Città di frontiera, città cerniera, Jaca Book, Milano (1997) 